\title{
An Improved Harmony Search Algorithm for Power Distribution Network Planning
}

\author{
Wei Sun and Xingyan Chang \\ Department of Business Administration, North China Electric Power University, Baoding 071003, China \\ Correspondence should be addressed to Xingyan Chang; changxi2013@163.com
}

Received 24 November 2014; Accepted 24 January 2015

Academic Editor: Ping Feng Pai

Copyright ( $) 2015$ W. Sun and X. Chang. This is an open access article distributed under the Creative Commons Attribution License, which permits unrestricted use, distribution, and reproduction in any medium, provided the original work is properly cited.

\begin{abstract}
Distribution network planning because of involving many variables and constraints is a multiobjective, discrete, nonlinear, and large-scale optimization problem. Harmony search (HS) algorithm is a metaheuristic algorithm inspired by the improvisation process of music players. HS algorithm has several impressive advantages, such as easy implementation, less adjustable parameters, and quick convergence. But HS algorithm still has some defects such as premature convergence and slow convergence speed. According to the defects of the standard algorithm and characteristics of distribution network planning, an improved harmony search (IHS) algorithm is proposed in this paper. We set up a mathematical model of distribution network structure planning, whose optimal objective function is to get the minimum annual cost and constraint conditions are overload and radial network. IHS algorithm is applied to solve the complex optimization mathematical model. The empirical results strongly indicate that IHS algorithm can effectively provide better results for solving the distribution network planning problem compared to other optimization algorithms.
\end{abstract}

\section{Introduction}

Distribution network planning can reduce the probability of blackouts, reduce transmission loss, and improve power quality, so that it is an important part of power distribution automation system. The main task of distribution network planning is to optimize network structure and find the optimal expansion scheme of power distribution network. The distribution network planning problem consists of minimizing investment and operation cost of the objective function subject to technical constraints, such as overload, voltage drop, and radial network. Distribution network expansion planning is a complex and large scale combinatorial optimization problem; the classical mathematical methods cannot perform satisfactorily for solving it [1-4]. Modern metaheuristic methods, such as Genetic Algorithm (GA), Particle Swarm Optimization (PSO), and Ant Colony System (ACS), are used recently to solve the distribution network planning problem and have achieved some results. However, the heuristic algorithms mentioned above have some defects: GA is a stochastic search algorithm for global optimization problems. It can reduce the difficulty of solving distribution network planning which is a nonlinear, multiconstraint, and multiobjective problem [5]. But in the practical application of the distribution network planning, it always falls into local optimum prematurely and converging slowly; there may even be infeasible solutions; PSO is a classic biological intelligence algorithm and has some advantages over other similar optimization techniques such as PSO which is easier to implement and there are fewer parameters to adjust, but it is prone to premature convergence [6]; ACS is a swarm intelligence algorithm based on distributed parallel search mechanism. It has strong robustness, but it also has some defects such as long calculation time, prone to stagnation and premature convergence $[7,8]$.

HS algorithm is a heuristic search algorithm for global optimization, which has been recently developed in an analogy with music improvisation process where musicians in an ensemble continue to polish their pitches in order to obtain better harmony [9]. The algorithm has the advantages of simple concept and model, easy implementation, less adjustable parameters, and quick convergence. However, when to solve 
the complex optimization problems, the standard HS algorithm still has some defects such as premature convergence. According to the defects of the standard algorithm and characteristics of distribution network planning, this paper proposed an improved harmony search (IHS) algorithm with the mechanism for dynamically adjusting parameters. We apply IHS algorithm to solve power distribution network planning. The simulation of example obviously shows that the solution is superior to that of other optimization algorithms.

\section{Mathematical Model of Distribution Network Planning}

In this paper, the model of distribution network planning adopts the objective function to minimize comprehensive costs per year, including the line investment expenses, the depreciation and maintenance costs, and the running year electrical energy loss expense. Considering overload constraints and radial network structure constraints, the objective function can be described as follows [10]:

$$
\min f(x)= \begin{cases}\sum_{i=1}^{n}\left(C_{1 i} T_{i} x_{i}+C_{2 i} \tau_{\max i} \Delta P_{i}\right)+M_{1} \times L, \\ \quad \text { when the network is radial } \\ M_{2}, \quad \text { else, }\end{cases}
$$

where $f(x)$ is the cost per year of distribution network planning; $C_{1 i}=\gamma_{i}+\partial_{i}, \gamma_{i}$ is the rate of return on investment; $\partial_{i}$ is annual depreciation rate of equipment; $T_{i}$ is the total investment for the $i$ th newly built line; $X$ is $n$-dimensional decision vectors; $X=\left(x_{1}, x_{2}, \ldots, x_{n}\right)$ is the $n$ transmission lines which is to be elected in the optimization problem. $x_{i}$ is the element of $X, x_{i}=1$ when the $i$ th line is newly built, and $x_{i}=0$ otherwise; $C_{2 i}$ is unit power price; $\tau_{\max i}$ is annual maximum load utilization hours; $\Delta P_{i}=\left(P_{i}^{2}+Q_{i}^{2}\right) R_{i} / U_{i}^{2}$ is the active power loss of the $i$ th line; $M_{1}$ is penalty coefficient of overload; $L$ is the load which exceeds the total load demand of power system; $M_{2}$ is a very large penalty value when the network is not radial.

\section{Harmony Search Algorithm}

Harmony search (HS) algorithm is a relatively new metaheuristic algorithm, which was proposed by Geem et al. [9]. Like other heuristic algorithms imitating natural phenomena or artificial ones, HS algorithm is also a heuristic algorithm mimicking the improvisation process of music players, where musicians improvise the pitches of their instruments to search for a perfect state of a harmony.

In HS algorithm, musical performances seek a perfect state of harmony determined by aesthetic estimation, as the optimization algorithms seek a global optimum determined by objective function value. Specifically, musical instrument $i(i=1,2, \ldots, n)$ is analogous to the $i$ th decision vector of the optimization problems. Each tone of the instrument is analogous to each value of the decision variable. The harmony $H_{j}(j=1,2, \ldots, n)$ produced by musical instruments is analogous to the $j$ th solution of the optimization problems.
Aesthetic evaluation is analogous to the objective function. The main control parameters of HS algorithm are harmony memory (HM), Harmony Memory Size (HMS), Harmony Memory Considering Rate (HMCR), Pitch Adjusting Rate (PAR), and Band Width (BW). Here, HM is a memory location where all the solution vectors are stored; HMCR and PAR are parameters that are used to improve the solution vector [11].

In the process of iteration, each new harmony vector $x^{\text {new }}=\left(x_{1}^{\text {new }}, x_{2}^{\text {new }}, \ldots, x_{n}^{\text {new }}\right)$ is generated based on three rules: (i) memory consideration, (ii) pitch adjustment, and (iii) random selection. Generating a new harmony is called "improvisation" [11]. In the memory consideration, the value of each component is updated as follows:

$$
x_{i}^{\text {new }}= \begin{cases}x_{i}^{j}, & j \in(1,2, \ldots, \text { HMS }), \operatorname{rand}_{1}<\text { HMCR } \\ x_{i} \in X_{i}, & \text { else }\end{cases}
$$

where rand $_{1}$ is a random number uniformly distributed in the range of $[0,1]$ and $X_{i}$ is the value space of the $i$ th variable.

Each component obtained by the memory consideration is examined to determine whether it should be pitchadjusted. The PAR parameter is the rate of pitch-adjustment. The equation of pitch-adjustment can be described as follows:

$$
x_{i}^{\text {new }}=\left\{\begin{array}{l}
x_{i}^{j} \pm \operatorname{rand}_{2} * \mathrm{BW}, \\
\operatorname{rand}_{2}<\operatorname{PAR} \text { (when } x_{i} \text { is continuous) } \\
x_{i}(k+m), \\
\operatorname{rand}_{2}<\operatorname{PAR} \text { (when } x_{i} \text { is discrete) } \\
x_{i}^{\text {new }}, \quad \text { else, }
\end{array}\right.
$$

where rand $_{2}$ is a random number uniformly distributed in the range of $[0,1]$ and $m$ is a constant, which belongs to the $(-1,1)$.

\section{Improved Harmony Search Algorithm}

4.1. HMCR. HMCR $\in[0,1]$ determines whether the value of a decision variable is to be chosen from HM. In order to ensure that the algorithm can quickly find local optima in the early operation and the solutions obtained in the later are diverse, this paper adopts the following linear decreasing strategy to update HMCR [12]:

$$
\operatorname{HMCR}(t)=\mathrm{HMCR}_{\max }-\frac{\left(\mathrm{HMCR}_{\max }-\mathrm{HMCR}_{\min }\right) * t}{T_{\max }}
$$

where $t$ denotes iteration number; $T_{\max }$ is the maximum total number of iterations; $\mathrm{HMCR}_{\max }$ and $\mathrm{HMCR}_{\min }$ represent maximum and minimum harmony memory considering rate, respectively.

4.2. PAR. In HS algorithm, PAR plays a role in controlling local search. The appropriate PAR can avoid the search being trapped in local optimum effectively. Normally, the smaller 


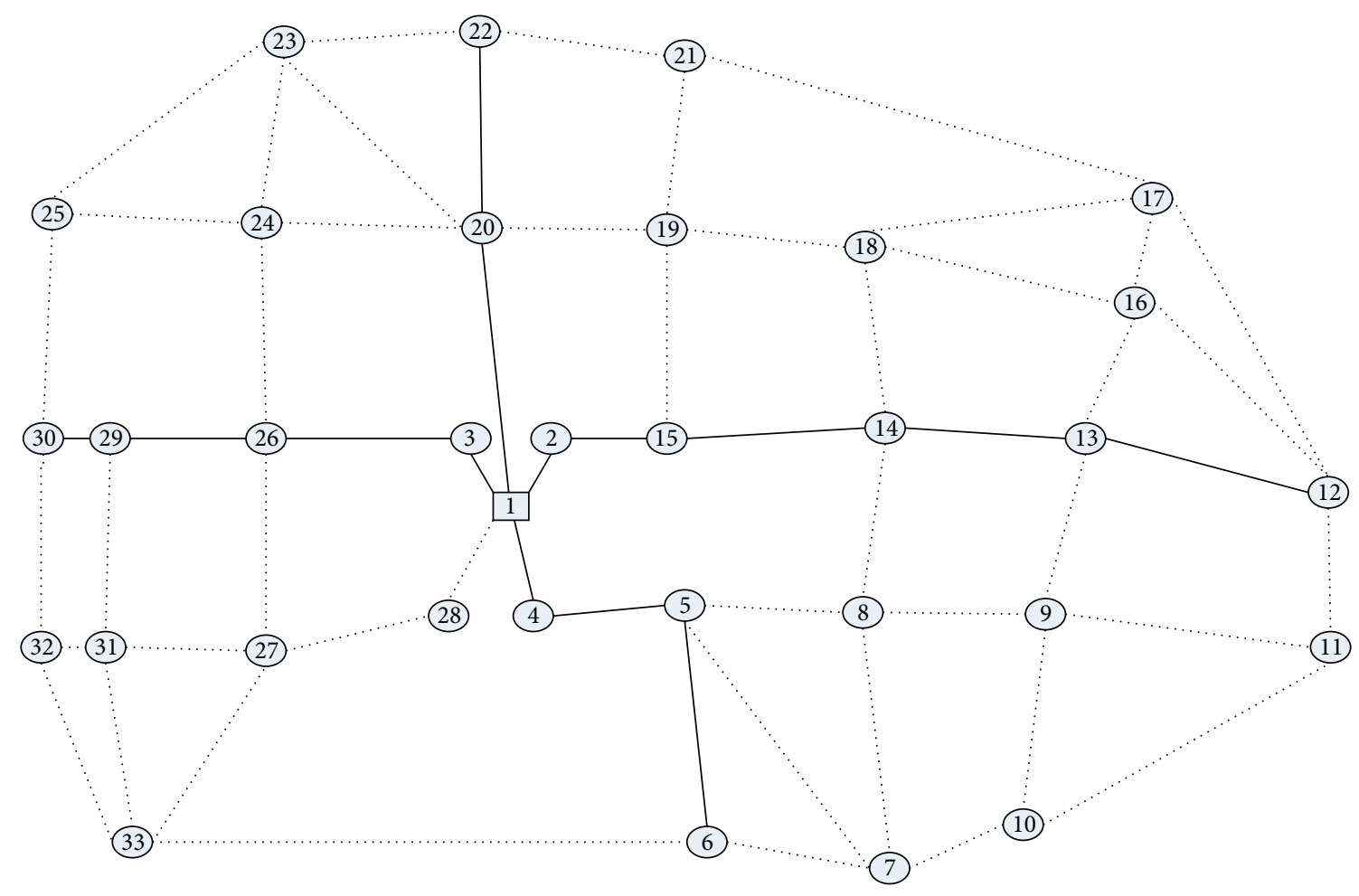

FIGURE 1: The existing network.

PAR is beneficial to quickly find the local optimal solution in early search stage, while the larger PAR is propitious to jump out the local optimal in the later stage. Therefore, dynamic change strategy for PAR is incorporated into the algorithm in this paper; the mathematical expression for PAR is

$$
\operatorname{PAR}(t)=\frac{\mathrm{PAR}_{\text {max }}-\mathrm{PAR}_{\text {min }}}{\pi / 2} * \arctan t+\mathrm{PAR}_{\text {min }},
$$

where $\operatorname{PAR}(t)$ denotes pitch adjusting rate for the $t$ th generation; $\mathrm{PAR}_{\max }$ and $\mathrm{PAR}_{\min }$ represent maximum and minimum harmony memory considering rate, respectively.

4.3. $B W$. The appropriate BW can be potentially useful in adjusting convergence rate of algorithm to optimal solution. In this paper, BW changes from large to small. BW changes dynamically with generation number as expressed as follows:

$$
\mathrm{BW}(t)=\mathrm{BW}_{\max }-\frac{\mathrm{BW}_{\text {max }}-\mathrm{BW}_{\text {min }}}{T_{\max }} * t,
$$

where $\operatorname{PAR}(t)$ denotes pitch band width for the $t$ th generation; $\mathrm{BW}_{\max }$ and $\mathrm{BW}_{\min }$ represent maximum and minimum harmony memory considering rate, respectively.

4.4. The Optimization Steps after Algorithm Improved. The optimization steps are summarized in the following.

Step 1. Initialize the HS algorithm parameters. Initialize the maximum number of iterations $T_{\max }$; the harmony memory size (HMS), the maximum and minimum harmony memory considering rate, $\mathrm{HMCR}_{\max }$ and $\mathrm{HMCR}_{\min }$; the maximum and minimum pitch adjusting rate, $\mathrm{PAR}_{\max }$ and $\mathrm{PAR}_{\min }$; the maximum and minimum band width, $\mathrm{BW}_{\max }$ and $\mathrm{BW}_{\min }$.

Step 2. Initialize the harmony memory (HM).

Step 3. Improvise a new harmony from the HM. Use (2), (3), (4), (5), and (6) to improvise a new harmony.

Step 4. Update the HM. Use (1) to evaluate fitness of the new harmony. If the new harmony is better than the worst harmony in the HM, the worst harmony is excluded from the $\mathrm{HM}$ and the new harmony is included in the HM.

Step 5. Inspect termination condition. The IHS will be terminated if the number of iterations meets the maximum number of iterations $T_{\max }$. Else go to Step 3 .

\section{Example Analysis}

In this paper, the proposed method for optimal distribution network planning is applied to a $10 \mathrm{kV}$ distribution system in a northern Chinese city using MATLAB 2011b. The network consists of a power supply point (110 KV substation), 32 load points, and 12 existing lines. 18 new load points are added to the existing network. The existing network is shown in Figure 1, in which the solid lines denote the existing lines and the dotted line denotes the expansible line. The coordinates and power of each load point are shown in Table 1. 


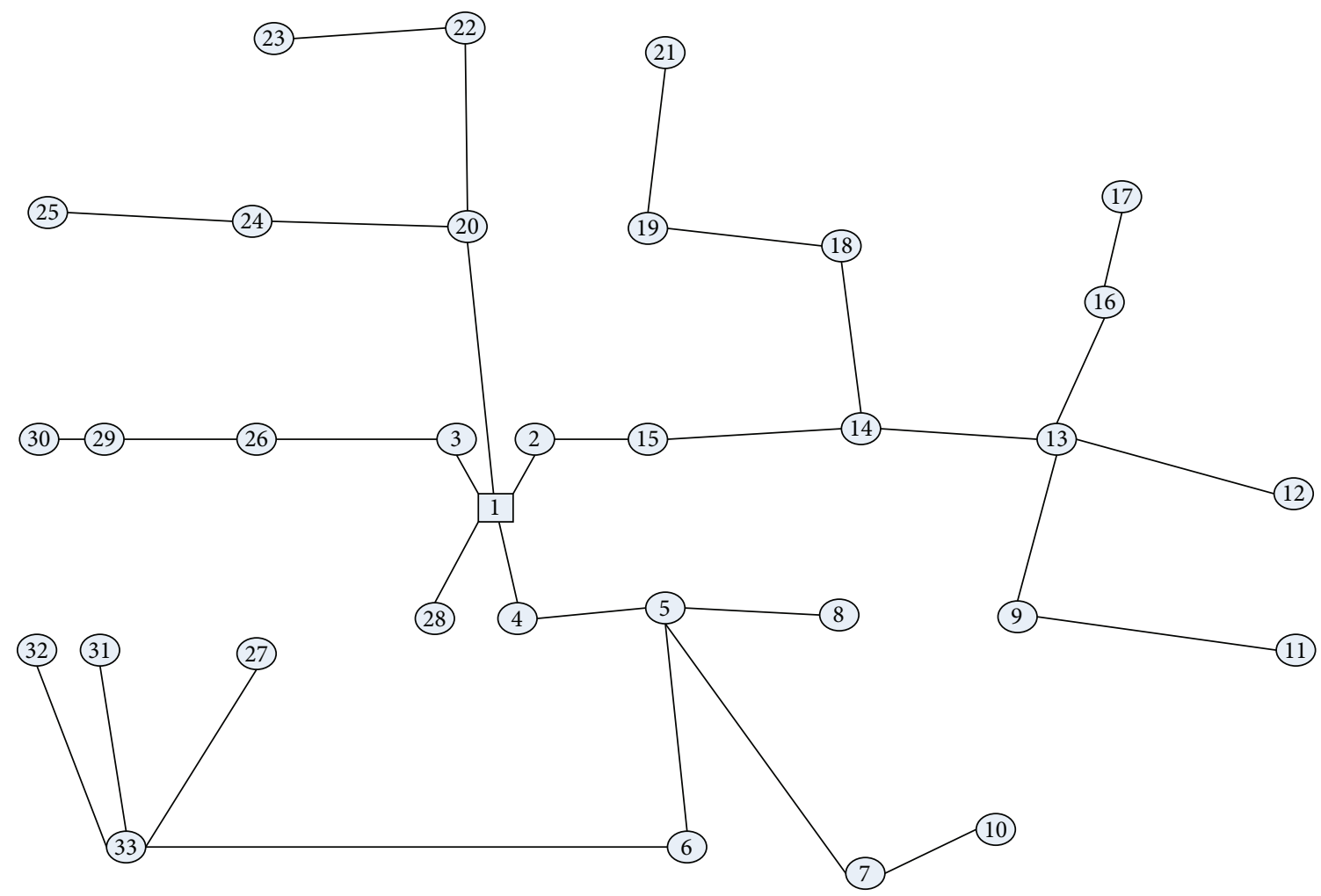

FIgURE 2: The optimal distribution network planning based on the HIS algorithm.

Using the improved algorithm to optimize the distribution network planning mentioned above, the input parameters are set as follows: $C_{1 i}=0.155, C_{2 i}=0.05$ yuan $/ \mathrm{KWh}$, $\tau_{\max i}=3000 \mathrm{~h}, \mathrm{HMS}=30, \mathrm{HMCR}_{\max }=0.95, \mathrm{HMCR}_{\min }=$ $0.6, \mathrm{PAR}_{\max }=0.99, \mathrm{PAR}_{\min }=0.01, \mathrm{BW}_{\max }=1.0, \mathrm{BW}_{\min }=$ 0.0001 , and $T_{\max }=1000$. The optimal distribution network planning based on the HIS algorithm is shown in Figure 2.

For further analysis, the IHS algorithm proposed in this paper, HS from [13], PSO from [14], Artificial Fish Swarm Algorithm (AFSA) from [15], Improved Ant Colony algorithm (IAC) from [16], Cross-Entropy Method (CE) [1719], and two typical evolutionary multiobjective optimization algorithms, Nondominated Sorting Genetic Algorithm version II (NSGA-II) [20-22] and Multiobjective Particle Swarm Optimization Algorithm (MOPSO) [23-25], are compared in optimizing the power network planning. In the case study, 50 independent runs were made for each of the optimization methods involving 50 different initial trial solutions for each optimization method. The parameters of each optimization method are shown in Table 2. The comparison results are shown in Table 3.

Comparing the results of Table 3, it can be found that the best solution (minimum cost) obtained by IHS algorithm is better than that of any other method. In terms of best solution and average optimal solution, it is very evident that the IHS algorithm proposed in this paper is superior to HS, PSO, AFSA, IAC, CE, NSGA-II, and MOPSO. This suggests that IHS algorithm has very strong stability and robustness. When to solve the multiobjective distribution network planning problem, the two typical evolutionary multiobjective optimization algorithms: NSGA-II and MOPSO are significantly superior to the HS, PSO, AFSA, IAC, and CE in terms of stability and robustness. But long execution times for these algorithms suggest that they reach the solution at a very slow speed. Though IHS algorithm obtained slightly better minimum cost and average cost than MOPSO, the average execution time used by IHS algorithm is less than that of MOPSO obviously. From the point of execution time, the average execution time of IHS algorithm is the minimum except CE. Although the execution time of CE is less than that of IHS algorithm, the best solution and average optimal solution obtained by the IHS algorithm are significantly better than that of CE. The reasonable average execution time of IHS algorithm suggests that IHS algorithm is capable of reaching the solution at a very high speed. Therefore, it can be concluded that IHS not only has found the highest quality results among all the algorithms compared, but also possesses greater stability and better robustness to solve such kinds of distribution network planning problem. IHS algorithm is an effective method to solve the distribution network planning problem.

\section{Conclusion}

Distribution network planning is a multiobjective, discrete, nonlinear, and large-scale optimization problem. This paper proposes an improved harmony search (IHS) algorithm to solve the distribution network planning problem. The 
TABLE 1: The coordinates and state of load points.

\begin{tabular}{|c|c|c|c|}
\hline Number & Abscissa (m) & Ordinate $(\mathrm{m})$ & Load (KVA) \\
\hline 1 & 2463.4 & 801.7 & substation \\
\hline 2 & 2462.5 & 808.4 & 500 \\
\hline 3 & 2457.1 & 807.9 & 400 \\
\hline 4 & 2460.6 & 773.3 & 200 \\
\hline 5 & 2481.4 & 775.3 & 600 \\
\hline 6 & 2477.5 & 728.8 & 800 \\
\hline 7 & 2503.2 & 724.5 & 400 \\
\hline 8 & 2503.7 & 772.8 & 200 \\
\hline 9 & 2530.8 & 773.4 & 500 \\
\hline 10 & 2528.5 & 730.3 & 800 \\
\hline 11 & 2572.3 & 778 & 400 \\
\hline 12 & 2573.6 & 799.9 & 600 \\
\hline 13 & 2533.6 & 807.7 & 500 \\
\hline 14 & 2508.3 & 809.4 & 400 \\
\hline 15 & 2478.7 & 808.2 & 200 \\
\hline 16 & 2535.4 & 837.5 & 500 \\
\hline 17 & 2537.7 & 854.4 & 1000 \\
\hline 18 & 2507 & 844.7 & 400 \\
\hline 19 & 2478.5 & 849 & 500 \\
\hline 20 & 2455.2 & 849.4 & 800 \\
\hline 21 & 2481.4 & 871.9 & 600 \\
\hline 22 & 2455.9 & 873.5 & 600 \\
\hline 23 & 2434 & 873 & 200 \\
\hline 24 & 2433.5 & 850 & 200 \\
\hline 25 & 2404.2 & 851.1 & 1000 \\
\hline 26 & 2433.9 & 807.8 & 500 \\
\hline 27 & 2434.3 & 775.2 & 800 \\
\hline 28 & 2456.5 & 776.3 & 400 \\
\hline 29 & 2417.6 & 808.3 & 200 \\
\hline 30 & 2403.5 & 808 & 200 \\
\hline 31 & 2416.4 & 774.6 & 600 \\
\hline 32 & 2403.5 & 773.7 & 1000 \\
\hline 33 & 2421.3 & 729.7 & 200 \\
\hline
\end{tabular}

TABle 2: The parameters of each optimization method.

\begin{tabular}{ll}
\hline Algorithms & Parameters \\
\hline HS & HMS $=30, \mathrm{HMCR}=0.95, \mathrm{PAR}=0.05, \mathrm{BW}=0.06$ \\
PSO & $c_{1}=2, c_{2}=2, w_{\max }=0.9, w_{\min }=0.4$, population $=40$ \\
AFSA & Step $=0.3$, visual $=1.8, \delta=0.618, A_{q}=0.9, c=0.85, t_{0}=50$, population $=50$ \\
IAC & $C=0.5, a=1.003, \rho=0.7$, population $=300$ \\
CE & $\mathrm{P}=0.01$, tol $=0.15, p_{0}=0.5, N=50$ \\
NSGA-II & $p_{c}=0.8, p_{m}=0.33, \eta_{c}=2, \eta_{m}=20$, QUOTE $T_{\max }=500$, population $=200$ \\
MOPSO & $w_{0}=0.35, w_{1}=1.0, \beta=0.1, \gamma=0.6, N=100, T_{\max }=250$, population $=100$ \\
\hline
\end{tabular}

TABLE 3: The comparison results of distribution network planning for some optimization algorithm.

\begin{tabular}{|c|c|c|c|}
\hline Algorithms & Best solution $/ 10^{8} \$$ & Average optimal solution $/ 10^{8} \$$ & Average execution time/s \\
\hline HS & 1.1005 & 1.2061 & 70.83 \\
\hline PSO & 1.1090 & 1.2162 & 71.25 \\
\hline AFSA & 1.0746 & 1.1860 & 80.61 \\
\hline IAC & 1.0772 & 1.1560 & 65.34 \\
\hline $\mathrm{CE}$ & 1.1108 & 1.2307 & 53.05 \\
\hline NSGA-II & 1.0487 & 1.1452 & 108.32 \\
\hline MOPSO & 1.0441 & 1.1367 & 104.19 \\
\hline IHS & 1.0432 & 1.1358 & 54.58 \\
\hline
\end{tabular}


parameters of basic HS algorithm are specifically improved for distribution network planning problem. The improved method can improve the global search ability and prevent basic HS algorithm into a local optimum. And the improved algorithm has a fast calculation and a good convergence. The numerical example shows that HIS algorithm not only can obtain the highest quality results but also possesses greater stability and better robustness. It is obvious that IHS can acquire satisfactory solution for distribution network planning.

\section{Conflict of Interests}

The authors declare that there is no conflict of interests regarding the publication of this paper.

\section{References}

[1] J. Salehi and M.-R. Haghifam, "Long term distribution network planning considering urbanity uncertainties," International Journal of Electrical Power and Energy Systems, vol. 42, no. 1, pp. 321-333, 2012.

[2] M. Sedghi, M. Aliakbar-Golkar, and M.-R. Haghifam, "Distribution network expansion considering distributed generation and storage units using modified PSO algorithm," International Journal of Electrical Power and Energy Systems, vol. 52, no. 1, pp. 221-230, 2013.

[3] M. Sedghi and M. Aliakbar-Golkar, "Distribution network expansion using hybrid SA/TS algorithm," Iranian Journal of Electrical and Electronic Engineering, vol. 5, no. 2, pp. 122-130, 2009.

[4] Y. Yang, G. Wei, B. Zhou, and X. Zhang, "Distribution network planning based on fuzzy expected value model," Transactions of China Electrotechnical Society, vol. 26, no. 4, pp. 200-206, 2011.

[5] H. Xu, L. Xin, H. Wang, and N. Yang, "Distribution network planning based on improved genetic algorithm," Guangdong Electric Power, vol. 23, no. 6, pp. 6-9, 2010.

[6] X. Zhenxia and J. Gu, "Application of particle swarm optimization algorithm to distribution network planning," Relay, vol. 34, no. 6, pp. 29-33, 2006.

[7] Y. Liang and H. Guan, "Medium-voltage distribution network planning based on improved ant colony optimization integrated with spanning tree," Transactions of the Chinese Society of Agricultural Engineering, vol. 29, no. 1, pp. 143-148, 2013.

[8] W. Sun and T. Ma, "The power distribution network structure optimization based on improved ant colony algorithm," Journal of Intelligent and Fuzzy Systems, vol. 26, no. 6, pp. 2799-2804, 2014.

[9] Z. W. Geem, J. H. Kim, and G. V. Loganathan, "A new heuristic optimization algorithm: harmony search," Simulation, vol. 76, no. 2, pp. 60-68, 2001.

[10] Z.-G. Lu, J. Liu, J. Wu, and Y.-B. He, "Application of artificial fish swarm algorithm in power distribution network planning," High Voltage Engineering, vol. 34, no. 3, pp. 565-602, 2008.

[11] L. D. S. Coelho and V. C. Mariani, "An improved harmony search algorithm for power economic load dispatch," Energy Conversion and Management, vol. 50, no. 10, pp. 2522-2526, 2009.

[12] Y. Chen and Y. Gao, "Multi-objective self-adaptive harmony search algorithm," Computer Engineering and Applications, vol. 47, no. 31, pp. 108-111, 2011.
[13] W.-L. Xiang, M.-Q. An, Y.-Z. Li, R.-C. He, and J.-F. Zhang, "An improved global-best harmony search algorithm for faster optimization," Expert Systems with Applications, vol. 41, no. 13, pp. 5788-5803, 2014.

[14] D. Tang, Y. Cai, J. Zhao, and Y. Xue, "A quantum-behaved particle swarm optimization with memetic algorithm and memory for continuous non-linear large scale problems," Information Sciences, vol. 289, no. 24, pp. 162-189, 2014.

[15] X. Cheng, Q. Zhang, Z. Wang, and H. Zhao, "Distribution network structure planning based on AFSA," Relay, vol. 35, no. 21, pp. 34-38, 2007.

[16] W. Sun, W. Shang, and D. Niu, "Application of improved ant colony optimization algorithm in distribution network planning," Power System Technology, vol. 30, no. 15, pp. 85-89, 2006.

[17] C. Lu, Y. Lu, and J. Zha, "A cross-entropy method for solving 0-1 knapsack problem," Computer Simulation, vol. 24, no. 7, pp. 183-187, 2007.

[18] K. Chepuri and T. Homem-de-Mello, "Solving the vehicle routing problem with stochastic demands using the crossentropy method," Annals of Operations Research, vol. 134, pp. 153-181, 2005.

[19] I. Giagkiozis, R. C. Purshouse, and P. J. Fleming, "Generalized decomposition and cross entropy methods for many-objective optimization," Information Sciences, vol. 282, pp. 363-387, 2014.

[20] S. Kannan, S. Baskar, J. D. McCalley, and P. Murugan, "Application of NSGA-II algorithm to generation expansion planning," IEEE Transactions on Power Systems, vol. 24, no. 1, pp. 454-461, 2009.

[21] K. Deb, A. Pratap, S. Agarwal, and T. Meyanvan, "A fast and e litist multi-objective genetic algorithm: NSGA-11," IEEE Transactions on Evolutionary Computation, vol. 6, no. 2, pp. 182197, 2002.

[22] S. H. Yang and U. Natarajan, "Multi-objective optimization of cutting parameters in turning process using differential evolution and non-dominated sorting genetic algorithm-II approaches," International Journal of Advanced Manufacturing Technology, vol. 49, no. 5-8, pp. 773-784, 2010.

[23] C.-Y. Zhang, M.-Y. Chen, C.-Y. Luo, J.-Q. Zhai, and Y. Jiang, "Power system reactive power optimization based on multiobjective particle swarm algorithm," Power System Protection and Control, vol. 38, no. 20, pp. 153-158, 2010.

[24] C. A. Coello Coello, G. T. Pulido, and M. S. Lechuga, "Handling multiple objectives with particle swarm optimization," IEEE Transactions on Evolutionary Computation, vol. 8, no. 3, pp. 256-279, 2004.

[25] M. A. Abido, "Multiobjective particle swarm optimization for optimal power flow problem," in Proceedings of the Optimization Power System Conference, vol. 8, pp. 241-268, Aswan, Egypt, 2010. 

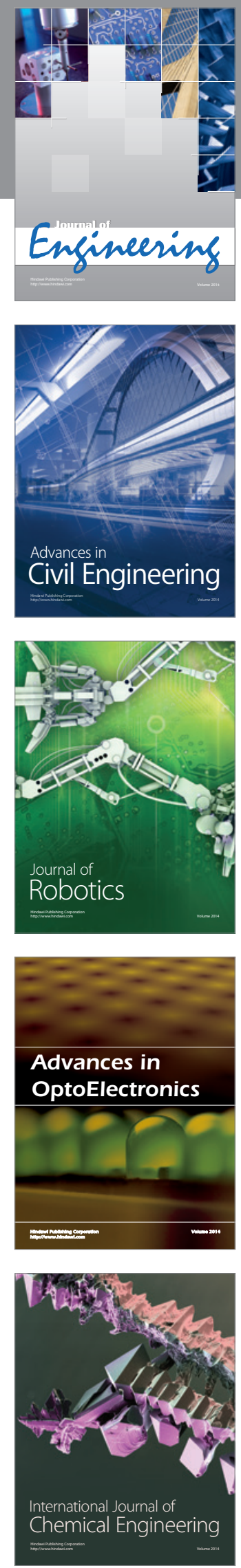

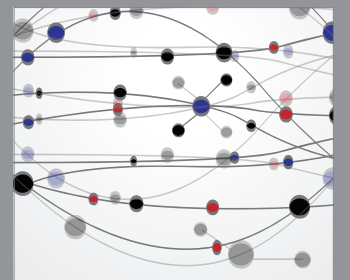

The Scientific World Journal
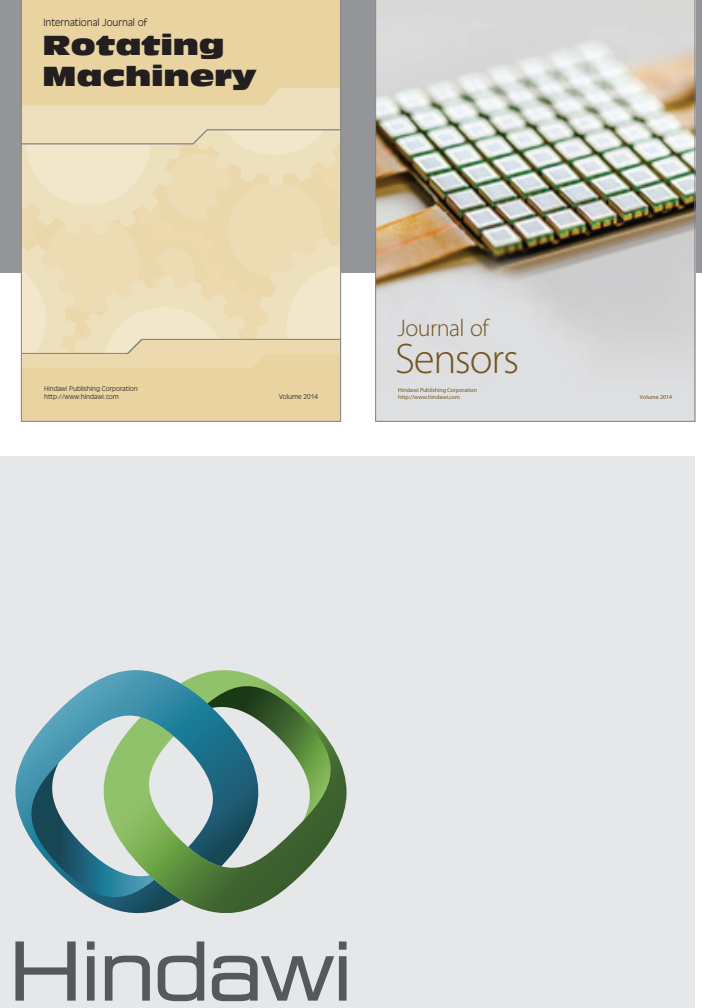

Submit your manuscripts at http://www.hindawi.com
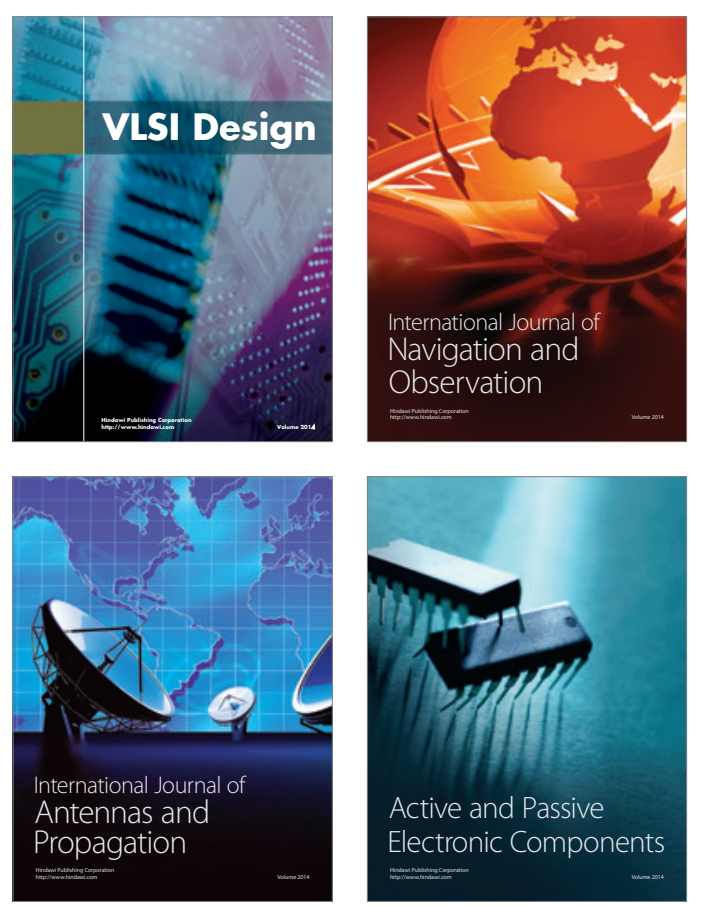
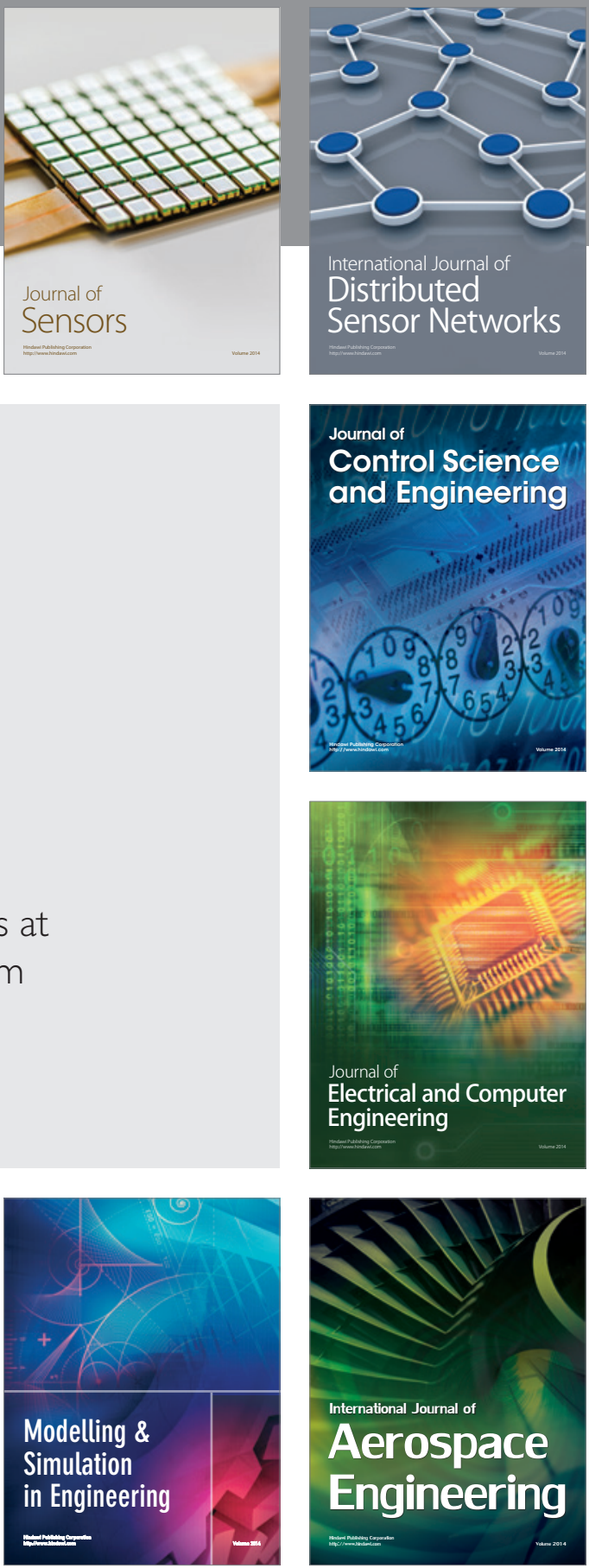

Journal of

Control Science

and Engineering
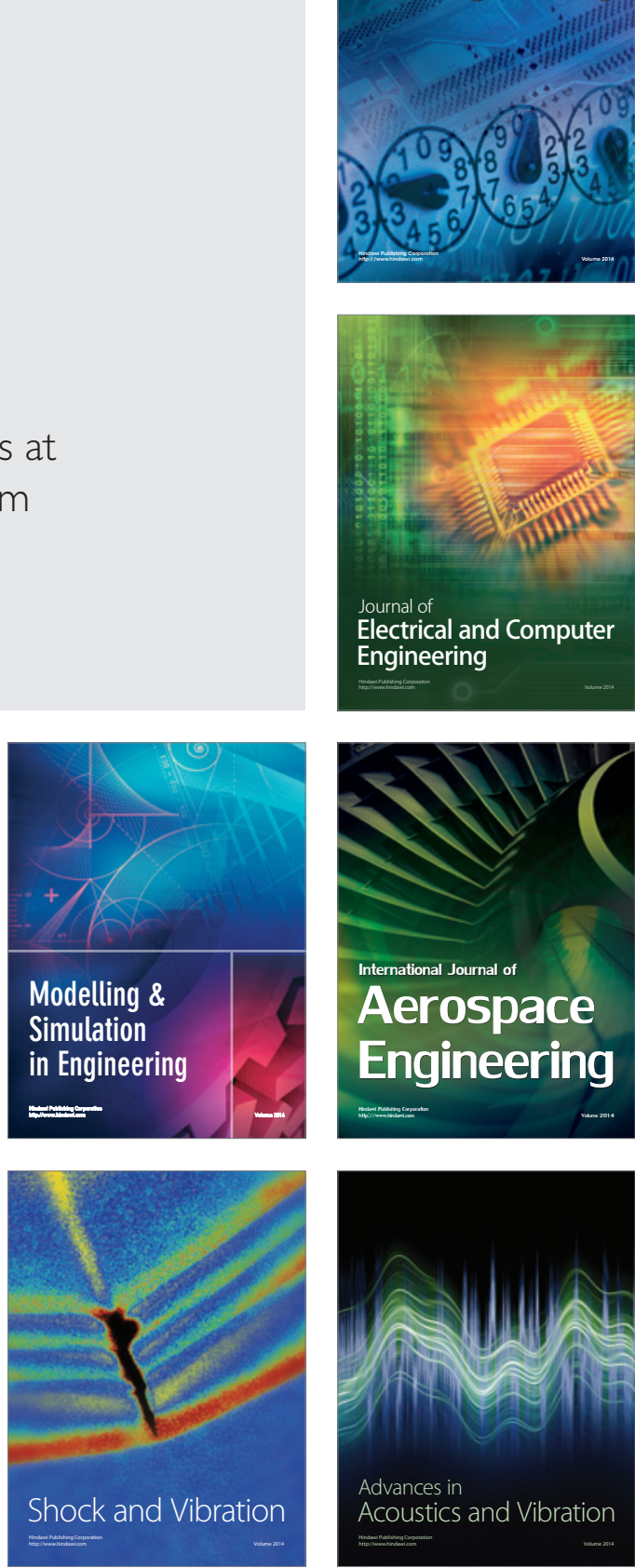